\title{
Automated lung sound analysis using the LungPass platform: a sensitive and specific tool for identifying lower respiratory tract involvement in COVID-19
}

\begin{abstract}
To the Editor:
Copyright @The authors 2021

This version is distributed under the terms of the Creative Commons Attribution Non-Commercial Licence 4.0. For commercial reproduction rights and permissions contact permissions@ersnet.org

Received: 7 July 2021

Accepted: 5 Sept 2021
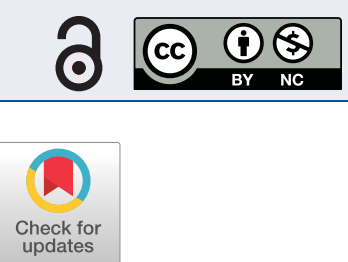

Lower respiratory tract (LRT) involvement, observed in about $20 \%$ of patients suffering from coronavirus disease 2019 (COVID-19), is associated with a more severe clinical course, adverse outcomes and long-term sequelae $[1,2]$. By pointing out people at risk of deterioration, early identification of LRT involvement could facilitate targeted and timely administration of treatments that could alter short- and long-term disease outcomes [3]. While imaging represents the gold standard diagnostic test for LRT involvement, it is associated with a potentially avoidable radiation burden and may not be easily accessible in some treatment settings, such as primary care [4]. Alternatively, oxygen desaturation appears to be a specific, but not sensitive marker, since ground glass changes or consolidation are often observed in the absence of hypoxia [5-7]. The sensitivity of chest auscultation in identifying LRT involvement has been evaluated in limited populations and varies $[8,9]$, possibly to some extent due to variable skill among the assessors.

The LungPass, an automated lung sound analysis platform consisting of an electronic wireless stethoscope paired with a mobile phone application, could standardise the auscultation process by limiting observer bias. The LungPass algorithm was developed using neural network technology and trained using sequential derivation sets of lung sound recordings. Next, the performance properties of the algorithm were evaluated in a separated validation cohort of 200 sound recordings. All the lung sound recordings used were adjudicated by a panel of expert respiratory physicians. Based on the validation cohort, the LungPass can identify normal lung sounds with a sensitivity of $96.9 \%$ and a specificity of $90 \%$, crackles $(92.5 \%, 82.5 \%)$ and wheeze (99.4\%, 90.0\%), as well as identifying artefacts and heart sounds (the development process will be reported separately).

We hypothesised that the LungPass could be used as a screening tool for LRT involvement in patients with COVID-19. In a prospective observational study that was conducted in the 5th City Clinical Hospital and the Minsk Regional Tuberculosis Dispensary (Minsk Region, Belarus), we evaluated the sensitivity of lung sounds assessed by a respiratory physician or by the LungPass in identifying LRT involvement in patients with COVID-19. The study included 282 adults presenting in the emergency department with a strong clinical suspicion of COVID-19 and imaging findings consistent with COVID-19 LRT involvement (ground glass opacities and/or consolidation). A confirmatory PCR result was available in $72.3 \%$ of the participants. Sequential chest auscultation in 11 pre-specified points (figure 1) was conducted using the LungPass followed by a consultant chest physician using a high-quality stethoscope, on the same occasion, and within $24 \mathrm{~h}$ from hospital presentation of the participants. The consultant chest physicians had extensive expertise in assessing patients with respiratory diseases (V.V. Khatsko, N.A. Voronova, M.V. Chamko; 5-20 years of fulltime clinical practice). Lung sounds in each auscultation site were recorded as normal breathing sounds, crackles, wheeze, cardiac sounds or artefacts (the last two types of sounds were excluded). To estimate the performance characteristics of the LungPass (sensitivity and specificity), we also used it to auscultate 32 consecutive adult patients admitted to the hospital with non-respiratory problems. The study was approved by the Belarusian State Medical Academy of Postgraduate Education ethics committee (ethics committee reference number: 18/21012020) at its meeting held on 6 February 2020 (protocol number 1/2020).

Shareable abstract (@ERSpublications)

Automated lung sound analysis using the \#LungPass platform is a sensitive and specific tool for identifying lower respiratory tract involvement in COVID-19 https://bit.ly/3tyAgOD

Cite this article as: Lapteva EA, Kharevich ON, Khatsko VV, et al. Automated lung sound analysis using the LungPass platform: a sensitive and specific tool for identifying lower respiratory tract involvement in COVID-19. Eur Respir J 2021; 58: 2101907 [DOI: 10.1183/13993003.01907-2021].
\end{abstract}




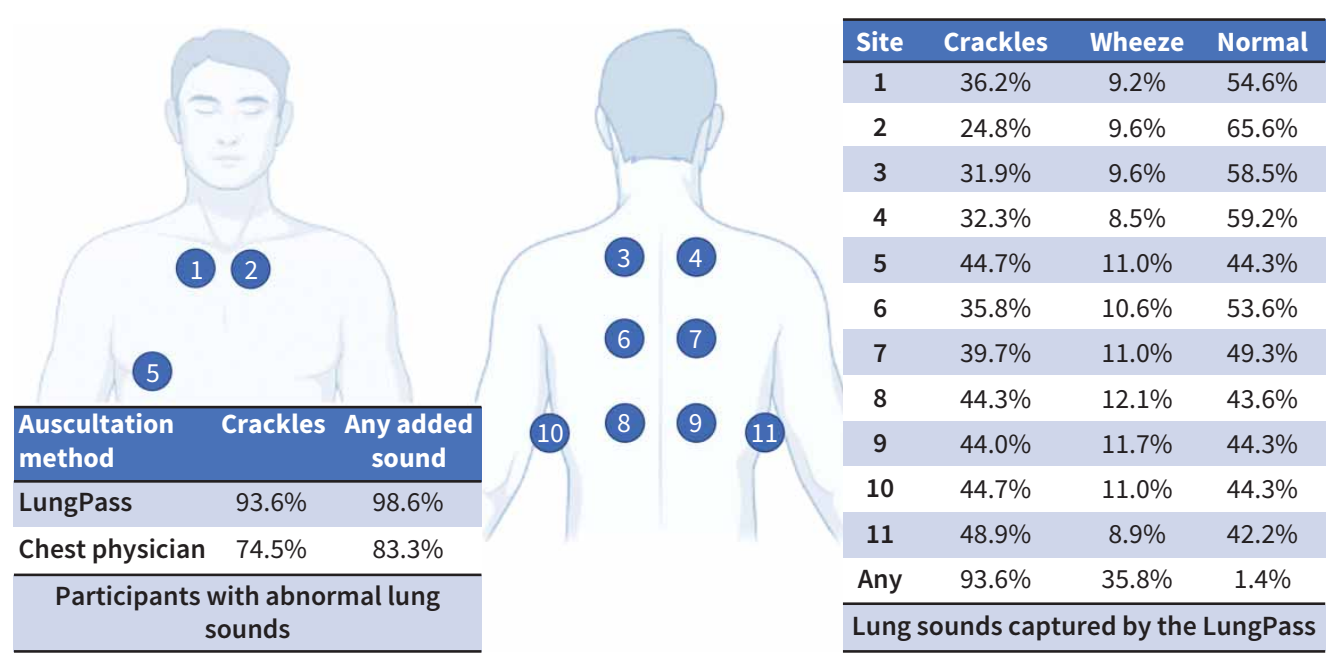

FIGURE 1 Auscultation points captured by the LungPass and chest physicians and the respective findings from participants with COVID-19 and lower respiratory tract involvement.

The mean \pm SD age of the participants in the COVID-19 group was $60.2 \pm 14.5$ years and $56 \%$ were females. Pre-existing chronic respiratory disorders were reported by $9.2 \%$ of these participants (mostly COPD, 6.7\%). The median (interquartile range) duration of symptoms at recruitment was 6 days (3-7 days). Most frequent presenting symptoms included fatigue (69.9\% of the participants), fever (66.7\%), cough (63.8\%) and breathlessness (35.8\%). Hypoxia (oxygen saturation less than 94\%) was observed only in $25.5 \%$ of the participants, despite all having radiographic evidence of COVID-19, including ground glass changes (97.9\%) and/or consolidation (20.2\%).

Overall, crackles were observed more frequently in the lower respiratory fields (figure 1). Cohen’s kappa index revealed a moderate-good agreement between the LungPass and respiratory physicians' judgements (kappa index 0.61, 95\% CI 0.59-0.64). Moreover, the agreement between the two more recently qualified chest physicians and the LungPass was better $(\mathrm{p}<0.001)$. The LungPass identified crackles in at least one auscultation site in $93.6 \%$ and in two or more points in $84.0 \%$ of the participants with COVID-19. The respective figures for respiratory physicians were $74.5 \%$ and $67.7 \%$. Moreover, the LungPass identified any added abnormal lung sounds (crackles or wheeze) in at least one auscultation site in $98.6 \%$ and in at least two points in $94.0 \%$ of the participants, while the respective percentages for the respiratory physician were $83.3 \%$ and $79.1 \%$.

Among the 3102 auscultation sounds (11 points in 282 patients), 533 (17.2\%) were deemed abnormal by the LungPass, but normal by the chest physician, while the opposite finding was observed for 175 (5.6\%) sounds. Interestingly, abnormal lung sounds were identified in one of the two most proximal, ipsilateral auscultation points in $73.6 \%$ and $86.3 \%$ of cases in each of the previously described groups, suggesting that the divergence between clinician and LungPass characterisation of the lung sounds is more likely to represent limited sensitivity to added abnormal lung sounds (false negatives), or differences in the positioning of the stethoscope, rather than limited specificity. For 1075 lung sounds that were deemed abnormal both by the clinician and LungPass, there was agreement in $97 \%$ of cases regarding the type of the abnormality (crackles or wheeze).

The mean \pm SD age of the control participants was $46.1 \pm 18.1$ years, $34.4 \%$ were females and none had underlying chronic respiratory conditions. The LungPass only identified abnormal lung sounds (crackles or wheeze) in one of the control patients, in two auscultation points. Based on our data, if the presence of any added, abnormal sound was considered evidence of LRT involvement, LungPass would have a sensitivity of $98.6 \%$ (95\% CI 96.4-99.6\%) and a specificity of 96.9\% (95\% CI 83.8-99.9\%). However, our previous data have suggested the presence of some false positive lung sound readings. To limit the risk of false positive readings, we could therefore consider the presence of any added abnormal lung sounds in at least two auscultation points as evidence of LRT involvement and, in this case, the sensitivity of LungPass is estimated to be $94.0 \%$ (95\% CI 90.5-96.5\%) and the specificity 96.9\% (95\% CI 83.8-99.9\%).

All results remained unchanged in a sensitivity analysis only including patients with COVID-19 confirmed by a positive PCR. 
These findings suggest the LungPass is a sensitive and specific platform for identifying LRT involvement due to COVID-19, even before the development of hypoxia. However, a limitation of this exploratory study was the control group consisting of patients without any acute respiratory problem, rather than patients with confirmed COVID-19 without LRT involvement (with clear chest computed tomography). Unfortunately, we did not have access to such individuals, since only patients with more severe COVID-19 reached the secondary care setting where this study was conducted during the COVID-19 pandemic. Therefore, future studies are needed to assess the performance characteristics of the LungPass for COVID-19 in a primary care setting.

Several lung sound recording devices have been used to facilitate auscultation during the COVID-19 pandemic without compromising clinicians' personal protective equipment [7, 8, 10]. Moreover, an extensive observational study evaluating the sensitivity and specificity of a different automated lung sound analysis algorithm is ongoing [11]. However, to our knowledge, this is the first exploratory study demonstrating the sensitivity and specificity of an automated lung sound analysis algorithm (the LungPass platform) in identifying LRT involvement in COVID-19. Upon confirmation of our findings in larger, ongoing studies, the LungPass, an affordable, portable lung sound recording device that could be used by non-experts, could facilitate telemonitoring and early identification of patients at risk of deterioration and, possibly, guide timely administration of potentially life-saving treatments.

Elena A. Lapteva ${ }^{1}$, Olga N. Kharevich ${ }^{1}$, Victoria V. Khatsko ${ }^{2}$, Natalia A. Voronova ${ }^{3}$, Maksim V. Chamko ${ }^{2}$, Irina V. Bezruchko ${ }^{4}$, Elena I. Katibnikova ${ }^{1}$, Elena I. Loban ${ }^{5}$, Mostafa M. Mouawie ${ }^{6}$, Helena Binetskaya ${ }^{6}$, Sergey Aleshkevich ${ }^{6}$, Aleksey Karankevich ${ }^{6}$, Vitaly Dubinetski ${ }^{6}$, Jørgen Vestbo $\oplus^{7,8}$ and Alexander G. Mathioudakis $\circledast^{7,8}$

${ }^{1}$ Belarusian State Medical Academy of Postgraduate Education, Minsk, Belarus. ${ }^{2} 5$ th City Clinical Hospital, Minsk, Belarus. ${ }^{3}$ Minsk Regional Tuberculosis Dispensary, Minsk Region, Belarus. ${ }^{4}$ Minsk Regional Pediatric Clinical Hospital, Minsk Region, Belarus. ${ }^{5}$ Minsk Clinical Center of Phthisiopulmonology, Minsk, Belarus. ${ }^{6}$ Healthy Networks OU, Tallinn, Estonia. ${ }^{7}$ Division of Infection, Immunity and Respiratory Medicine, School of Biological Sciences, The University of Manchester, Manchester Academic Health Science Centre, Manchester, UK. ${ }^{8}$ The North West Lung Centre, Wythenshawe Hospital, Manchester University NHS Foundation Trust, Manchester, UK.

Corresponding author: Alexander G. Mathioudakis (a.mathioudakis@nhs.net)

Author contributions: Design: E.A. Lapteva, O.N. Kharevich, H. Binetskaya and A.G. Mathioudakis; data collection: E.A. Lapteva, O.N. Kharevich, V.V. Khatsko, N.A. Voronova, M.V. Chamko, I.V. Bezruchko, E.I. Katibnikova and E.I. Loban; data processing: E.A. Lapteva, O.N. Kharevich, I.V. Bezruchko, E.I. Katibnikova, E.I. Loban, M.M. Mouawie, S. Aleshkevich, A. Karankevich and V. Dubinetski; data analysis: O.N. Kharevich and A.G. Mathioudakis; manuscript preparation: A.G. Mathioudakis; critical review of the manuscript: all authors; data guarantors: E.A. Lapteva, O.N. Kharevich and H. Binetskaya.

Conflict of interest: E.A. Lapteva is an employee of and holds shares in Healthy Networks, and reports personal fees from Healthy Networks, outside the submitted work. O.N. Kharevich is an employee of Healthy Networks, and reports personal fees from Healthy Networks, outside the submitted work. V.V. Khatsko reports personal fees from Healthy Networks, during the conduct of the study. N.A. Voronova reports personal fees from Healthy Networks, during the conduct of the study. M.V. Chamko reports personal fees from Healthy Networks, during the conduct of the study. I.V. Bezruchko reports personal fees from Healthy Networks, during the conduct of the study; personal fees from Healthy Networks, outside the submitted work. E.I. Katibnikova reports personal fees from Healthy Networks, during the conduct of the study; personal fees from Healthy Networks, outside the submitted work. E.I. Loban is an employee of Healthy Networks, and reports personal fees from Healthy Networks, outside the submitted work. M.M. Mouawie is an employee of Healthy Networks. H. Binetskaya is the CEO of and holds shares in Healthy Networks, and holds a patent on Lung Sound Classification Software (pending). S. Aleshkevich is employed by Healthy Networks. A. Karankevich is employed by and holds shares in Healthy Networks, and holds a patent on Lung Sound Classification Software (pending). V. Dubinetski is employed by and holds shares in Healthy Networks, and holds a patent on Lung Sound Classification Software (pending). J. Vestbo reports personal fees for consultancy and lectures from AstraZeneca, Chiesi and Novartis, grants and personal fees for consultancy and lectures from Boehringer Ingelheim, personal fees for consultancy from GSK, outside the submitted work; and his son works for Chiesi. A.G. Mathioudakis holds shares in Healthy Networks, and reports grants from Boehringer Ingelheim, outside the submitted work. 
Support statement: This study was funded and sponsored by Healthy Networks. J. Vestbo and A.G. Mathioudakis are supported by the National Institute for Health Research Manchester Biomedical Research Centre (NIHR Manchester BRC). Funding information for this article has been deposited with the Crossref Funder Registry.

\section{References}

1 Mason RJ. Pathogenesis of COVID-19 from a cell biology perspective. Eur Respir J 2020; 55: 2000607.

2 Sonnweber T, Sahanic S, Pizzini A, et al. Cardiopulmonary recovery after COVID-19: an observational prospective multicentre trial. Eur Respir J 2021; 57: 2003481.

3 Mathioudakis AG, Fally M, Hashad R, et al. COVID-19 clinical trials: unraveling a methodological Gordian knot. Am J Respir Crit Care Med 2020; 202: 635-637.

4 Morikawa M, Shinoda M, Ota S, et al. Clinical features of 154 COVID-19 patients and the parameters for the effective detection of pneumonia at the time of the initial diagnosis in Japan. Intern Med 2021; 60: 31-37.

5 Marco CA, Repas SJ, Studebaker H, et al. Radiographic findings of SARS-CoV-2 infection. J Am Coll Emerg Physicians Open 2021; 2: e12399.

6 Osman AM, Farouk S, Osman NM, et al. Longitudinal assessment of chest computerized tomography and oxygen saturation for patients with COVID-19. Egypt J Radiol Nucl Med 2020; 51: 255.

7 Zieleskiewicz L, Markarian T, Lopez A, et al. Comparative study of lung ultrasound and chest computed tomography scan in the assessment of severity of confirmed COVID-19 pneumonia. Intensive Care Med 2020; 46: $1707-1713$.

8 Wang B, Liu Y, Wang Y, et al. Characteristics of pulmonary auscultation in patients with 2019 novel coronavirus in China. Respiration 2020; 99: 755-763.

9 Zhang P, Wang B, Liu Y, et al. Lung auscultation of hospitalized patients with SARS-CoV-2 pneumonia via a wireless stethoscope. Int J Med Sci 2021; 18: 1415-1422.

10 Yang C, Zhang W, Pang Z, et al. A low-cost, ear-contactless electronic stethoscope powered by Raspberry Pi for auscultation of patients with COVID-19: prototype development and feasibility study. JMIR Med Inform 2021; 9: e22753.

11 Glangetas A, Hartley MA, Cantais A, et al. Deep learning diagnostic and risk-stratification pattern detection for COVID-19 in digital lung auscultations: clinical protocol for a case-control and prospective cohort study. BMC Pulm Med 2021; 21: 103. 